\title{
Influence of Physiological Noise on Accelerated 2D and 3D Resting State Functional MRI Data at $7 \mathrm{~T}$
}

\author{
Olivier Reynaud, ${ }^{1 *}$ João Jorge $\mathbb{D}^{0},{ }^{2}$ Rolf Gruetter, ${ }^{1,2,3,4}$ José P. Marques, ${ }^{1,5}$ and \\ Wietske van der Zwaag ${ }^{1,6}$
}

Purpose: Physiological noise often dominates the bloodoxygen level-dependent (BOLD) signal fluctuations in high-field functional MRI (fMRI) data. Therefore, to optimize fMRI protocols, it becomes crucial to investigate how physiological signal fluctuations impact various acquisition and reconstruction schemes at different acquisition speeds. In particular, further differences can arise between 2D and 3D fMRI acquisitions due to different encoding strategies, thereby impacting fMRI sensitivity in potentially significant ways.

Methods: The amount of physiological noise to be removed from the BOLD fMRI signal acquired at $7 \mathrm{~T}$ was quantified for different sampling rates (repetition time from 3300 to $350 \mathrm{~ms}$, acceleration 1 to 8 ) and techniques dedicated to fast fMRI (simultaneous multislice echo planar imaging [EPI] and 3D EPI). Resting state fMRI (rsfMRI) performances were evaluated using temporal signal-tonoise ratio (tSNR) and network characterization based on seed correlation and independent component analysis.

Results: Overall, acceleration enhanced tSNR and rsfMRI metrics. 3D EPI benefited the most from physiological noise removal at long repetition times. Differences between $2 \mathrm{D}$ and $3 \mathrm{D}$ encoding strategies disappeared at high acceleration factors (6- to 8-fold).

Conclusion: After physiological noise correction, 2D- and 3Daccelerated sequences provide similar performances at high fields, both in terms of tSNR and resting state network identification and characterization. Magn Reson Med 78:888-896, 2017. (C) 2017 International Society for Magnetic Resonance in Medicine.

Key words: controlled aliasing; 3D EPI; SMS; physiological noise; resting state fMRI; CAIPIRINHA

\section{INTRODUCTION}

Most functional MRI (fMRI) studies rely on echo planar imaging (EPI) sequences (1) to probe the brain's function with a high sampling rate compared with the hemodynamic

\footnotetext{
${ }^{1}$ Centre d'Imagerie Biomédicale, Ecole Polytechnique Fédérale de Lausanne, Lausanne, Switzerland.

${ }^{2}$ Laboratoire for Functional and Metabolic Imaging, Ecole Polytechnique Fédérale de Lausanne, Lausanne, Switzerland.

${ }^{3}$ Department of Radiology, University of Geneva, Geneva, Switzerland.

${ }^{4}$ Department of Radiology, University of Lausanne, Lausanne, Switzerland.

${ }^{5}$ Donders Institute for Brain Behaviour and Cognition, Nijmegen, Netherlands.

${ }^{6}$ Spinoza Centre for Neuroimaging, Amsterdam, Netherlands.

Grant sponsor: Centre d'Imagerie BioMédicale of the UNIL, UNIGE, HUG, CHUV, EPFL, and Leenaards and Jeantet Foundations.

*Correspondence to: Olivier Reynaud, Ph.D., Centre d'Imagerie Biomédicale, EPFL SB CIBM-AIT, CH F0 572 (Bâtiment CH), Station 6, CH-1015 Lausanne, Switzerland. E-mail: oli.reynaud@gmail.com

Received 28 March 2017; revised 6 June 2017; accepted 13 June 2017 DOI $10.1002 / \mathrm{mrm} .26823$

Published online 7 July 2017 in Wiley Online Library (wileyonlinelibrary.com).
}

(C) 2017 International Society for Magnetic Resonance in Medicine

response, usually $0.3-0.5 \mathrm{~Hz}$. For resting state fMRI (rsfMRI), better spatiotemporal characterization of the networks can be achieved through increases in temporal resolution. At ultrahigh fields ( $>7 \mathrm{~T}$ ), high spatial resolution is also desired because of reduced susceptibility artifacts in higher-resolution EPI and the improved spatial fMRI specificity. For equal brain coverage, both echo train length and slice numbers increase when spatial resolution increases, resulting in longer acquisition durations per volume.

The acquisition time is typically reduced by accelerating the EPI acquisition (2) using parallel imaging (3-5). The simultaneous multislice (SMS) technique, originally developed by Larkman et al. (6) and later adapted for EPI (7), excites several slices at once and separates the MRI signal using the receiver array information, reducing acquisition times up to one order of magnitude using commercial receiver coils. The standard SMS-EPI implementation now includes controlled aliasing using blipped gradients (8), effectively increasing the distance between superimposed voxels without through-slice blurring penalty and allowing even higher acceleration factors with relatively increased image quality. The benefits of using SMS for fMRI at $7 \mathrm{~T}$ are well documented $(9,10)$.

For 3D EPI, the readout duration necessary to encode the full volume with single-shot techniques $(11,12)$ can be very hardware-demanding and constrain the achievable echo time TE (otherwise matching $\mathrm{T}_{2}{ }^{*}$ for optimal BOLD contrast). By comparison, segmented 3D-EPI techniques $(13,14)$ acquire only one/several k-space planes after each slab excitation, so that multiple, shorter segments are required to reconstruct the whole volume and can be easily accelerated in one or two directions. Compared with SMS-EPI, 3D-EPI techniques stand to benefit from an increased signal-tonoise ratio (SNR) $(15,16)$ and the absence of spin history artifacts (17). Segmented 3D EPI using controlled aliasing was used to characterize the brain hemodynamic response at $400 \mathrm{~ms}$ temporal resolution with whole brain coverage (18).

Physiological signal fluctuations, often referred to as physiological noise, represent a major nuisance for activation detection using fMRI (19-21). Over the years, a large number of mechanisms have been identified as sources of physiological noise, including cardiac and respiratory cycles and depth rates. RETROICOR models (22) have been proposed to account for the former, namely phase-related contributions, whereas the latter can be modeled using cardiac rate (CR) (23-25) and respiratory volume per unit time (RVT) models $(26,27)$. At high fields, a combination of these various regressors has been reported to improve the temporal SNR (tSNR) 
$(19,21,28)$, a quantitative indicator of fMRI detection capabilities.

Multishot sequences are particularly prone to physiological noise, because different segments will be affected differently by respiration, cardiac rate, and more generally, motion. Physiological noise correction on 3D data led to small improvements in BOLD detection compared with standard 2D EPI for nonaccelerated 3D EPI at $7 \mathrm{~T}$ $(19,29)$ and accelerated 3D EPI at 3T $(20)$. Because segmented 3D EPI approaches are more susceptible to physiological fluctuations than $2 \mathrm{D}$ sequences, the advantage of using 3D accelerated sequences may not directly translate into a higher image SNR. This sensitivity to noise should decrease as the temporal resolution increases $(14,30)$ and is expected to become negligible for highly accelerated sequences. For SMS-EPI, several algorithms (8,31-35) have been proposed to reconstruct the accelerated data. However, the removal of physiological noise after different reconstructions has yet not been investigated, and should be characterized before comparing 2D and 3D techniques.

To determine which sequences are better suited to perform fMRI at high fields, the aim of the present study was to characterize physiological noise by investigating its impact on SMS data reconstructed using different reconstruction approaches, and the impact of acceleration factor and sequence type (2D versus $3 \mathrm{D} \mathrm{EPI)} \mathrm{on} \mathrm{physiological}$ noise correction and rsfMRI performances after noise removal.

\section{METHODS}

All experiments were performed on a Siemens 7 T, 68-cm head scanner (Siemens Healthcare, Erlangen, Germany) using a single-channel quadrature transmit and 32channel receive coil array (Nova Medical Inc., Wilmington, Massachusetts, USA) (36). All participants provided written informed consent prior to the experiments, which were conducted in agreement with procedures approved by an institutional review board.

\section{MRI Protocol}

Experiments were performed on eight healthy subjects ( $\mathrm{n}=5$ male; $\mathrm{n}=3$ female; age, $22 \pm 3$ years). For physiological noise correction, cardiac and respiration data were recorded at $50 \mathrm{~Hz}$ frequency using a vendor-provided plethysmograph and respiration belt, respectively. The duration of each resting state acquisition was $5 \mathrm{~min}$.

2D whole-brain rsfMRI data were acquired using SMSEPI (6) and the blipped-CAIPIRINHA method for controlled aliasing (8). The MR parameters were: echo time $=26 \mathrm{~ms}$, isotropic resolution $=2 \mathrm{~mm}$, matrix size $=106 \times 90 \times 54$, and partial Fourier encoding along the phase-encoding (PE) direction $=7 / 8$. The run was repeated using three repetition times $(\mathrm{TR})=3300 / 550 / 350 \mathrm{~ms}$ corresponding to different multiband $(\mathrm{MB})$ factors $=1 / 6 / 8$. Controlled aliasing was performed using a FOV/3 PE shift based on preliminary studies. Flip angles were adjusted to the Ernst angle corresponding to $\mathrm{T}_{1}=1800 \mathrm{~ms}$.

A segmented 3D EPI using controlled aliasing (18) was used for comparison. Sequence parameters were kept constant whenever possible. $\mathrm{TR}=3300 / 550 / 350 \mathrm{~ms}$ was achieved using acceleration factors along the second $\mathrm{PE}$ direction $\mathrm{Rz}=1 / 6 / 8$ with CAIPIRINHA shifts $\Delta=0 / 2 / 3$ (see Fig. 1a,b, sequence chronogram and k-space coverage with $\mathrm{Rz}=6$ and $\Delta=2$ ). Flip angles were adjusted to the duration of a single segment $\mathrm{TR}_{\text {segment }}=\mathrm{TR} / \mathrm{Rz}$. For generalized autocalibrating partially parallel acquisition (GRAPPA) (4) calibration, a set of reference lines (size $42 \times 42$ ) was acquired prior to the first volume. 3D EPI volumes were reconstructed offline using a 2D GRAPPA algorithm (37) in MATLAB (MathWorks, Natick, Massachusetts, USA).

The six 2D- and 3D-rsfMRI scans with different TRs were counter-balanced across subjects. After each run, six additional volumes were acquired with opposite PE direction (left to right) to correct for EPI distortions retrospectively using the topup toolbox in FSL (38).

\section{Reconstruction of SMS Data}

Because different approaches have been proposed to reconstruct SMS-EPI data, the resultant images could be impacted differently by physiological noise correction. Historically, the SENSE/GRAPPA formalism considered the undersampled set of excited slices (Fig. 2a) as a concatenation of the same slices along the PE direction (31). The introduction of shifted slices (necessary for controlled aliasing) resulted, however, in phase discontinuities and image artifacts. To overcome this problem, other methods were developed, including concatenation along the readout direction (35) (see Fig. 2b) or periodic zero-filling. Recently, the slice GRAPPA (SG) (8) or split-slice GRAPPA (SP-SG) (32) methods have adopted a different vision; for SG, GRAPPA is performed for each slice independently from the same undersampled dataset (Fig. 2c, as many GRAPPA kernels as there are slices); for SP-SG, an additional slice-dependent filter prevents signal leakage from the other excited slices. Finally, it was also shown that the SMS reconstruction problem could be represented by an equivalent 3D k-space slice-encoding problem (Fig. 2d) $(33,34)$, and reconstructed using standard 2D GRAPPA used for volumetric imaging (37) (Fig. 1b).

The 6-fold accelerated 2D SMS dataset was reconstructed here using 1) concatenation along the readout direction (35), 2) slice GRAPPA (8), and 3) the SMS equivalent 3D formalism $(33,34)$.

\section{Physiological Noise Characterization}

Physiological signals, mainly respiratory and cardiac signals, have been shown to impact the fMRI signal stability or tSNR. In this study, a conventional general linear model (GLM) regression approach was applied after data preprocessing including EPI distortion correction using topup (38), motion correction, and slice timing (for SMS). No smoothing was applied. The GLM design matrix included slow-drift signals (first-, second-, and third order polynomials), de-trended motion parameters (three rotations, three translations), and cardiac and respiratory parts of RETROICOR (RETc/RETr, up to second order), cardiac rate (CR) and respiratory volume per unit time (RVT). For CR and RVT, responses were respectively convolved with the cardiac and respiratory response functions detailed in $(24,27)$. For 3D EPI (no slice timing), regressors were synchronized with $\mathrm{TR} / 2$, corresponding to the k-space center encoding $(20,21)$. 


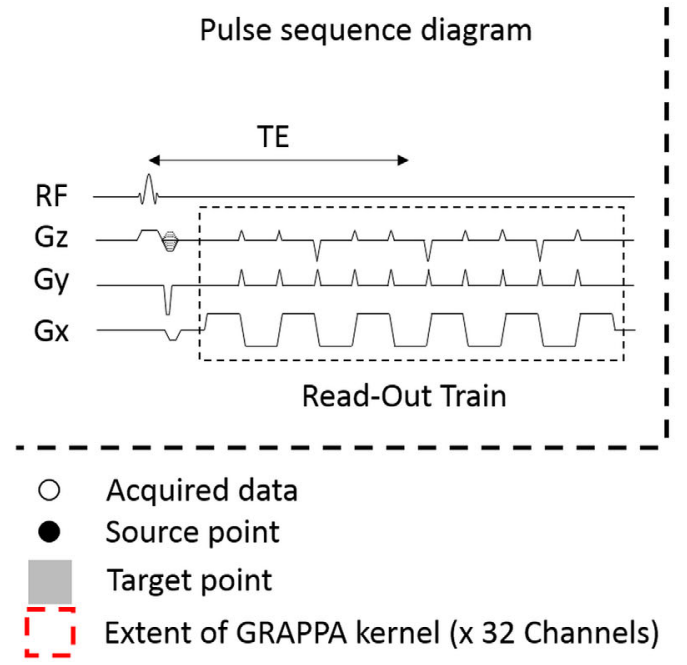

a

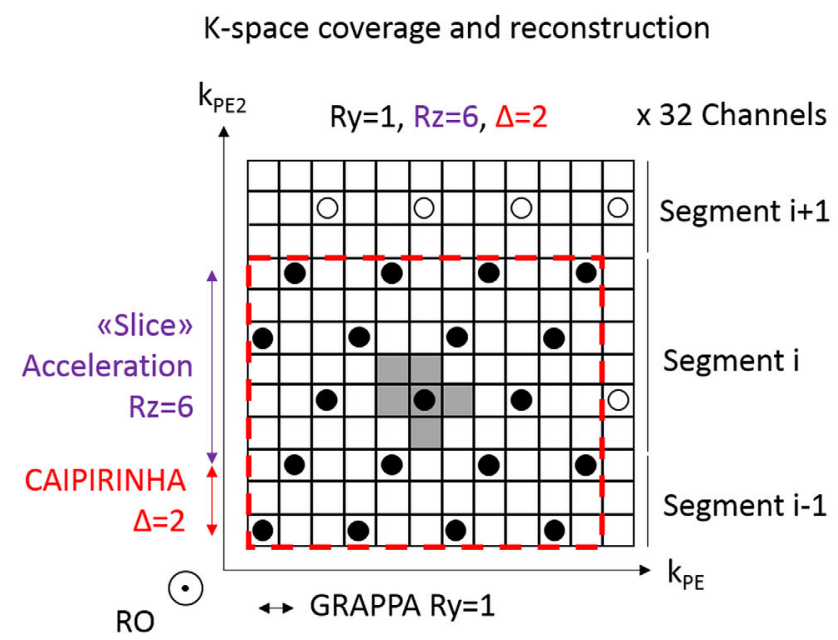

b

FIG. 1. (a) Pulse sequence diagram and (b) k-space for segmented 3D EPI using controlled aliasing (Ry=1, Rz=6, $\Delta=2)$. The black, purple, and red arrows indicate the impact of GRAPPA along PE, slice acceleration, and CAIPIRINHA FOV shift, respectively, on the k-space sampling pattern. The source points used to reconstruct the missing 3D data (in gray) are delineated by the dashed red rectangle. The whole k-space is reconstructed by shifting the corresponding GRAPPA kernel in 2D. GRAPPA, generalized autocalibrating partially parallel acquisition; RF, radiofrequency; TE, echo time.

The spatial contribution of the different regressors (drifts, motion, RETc, RETr, CR, RVT) was inspected visually using the absolute value of their respective GLM-based Z-scores.

After tissue segmentation using the FAST toolbox of FSL, the noise influence was characterized using the adjusted coefficient of determination $R_{\mathrm{adj}}^{2}$. This metric estimates how much signal can be explained by a particular set of noise regressors and is weighted by the number of these regressors, and therefore independent of the degrees of freedom number (25). The $\mathrm{R}_{\mathrm{adj}}^{2}$ of motion, slow signal drifts, respiratory and cardiac RETROICOR (RETr/RETc), RVT, and CR were compared for $\mathrm{TR}=3300 / 550 / 350 \mathrm{~ms}$

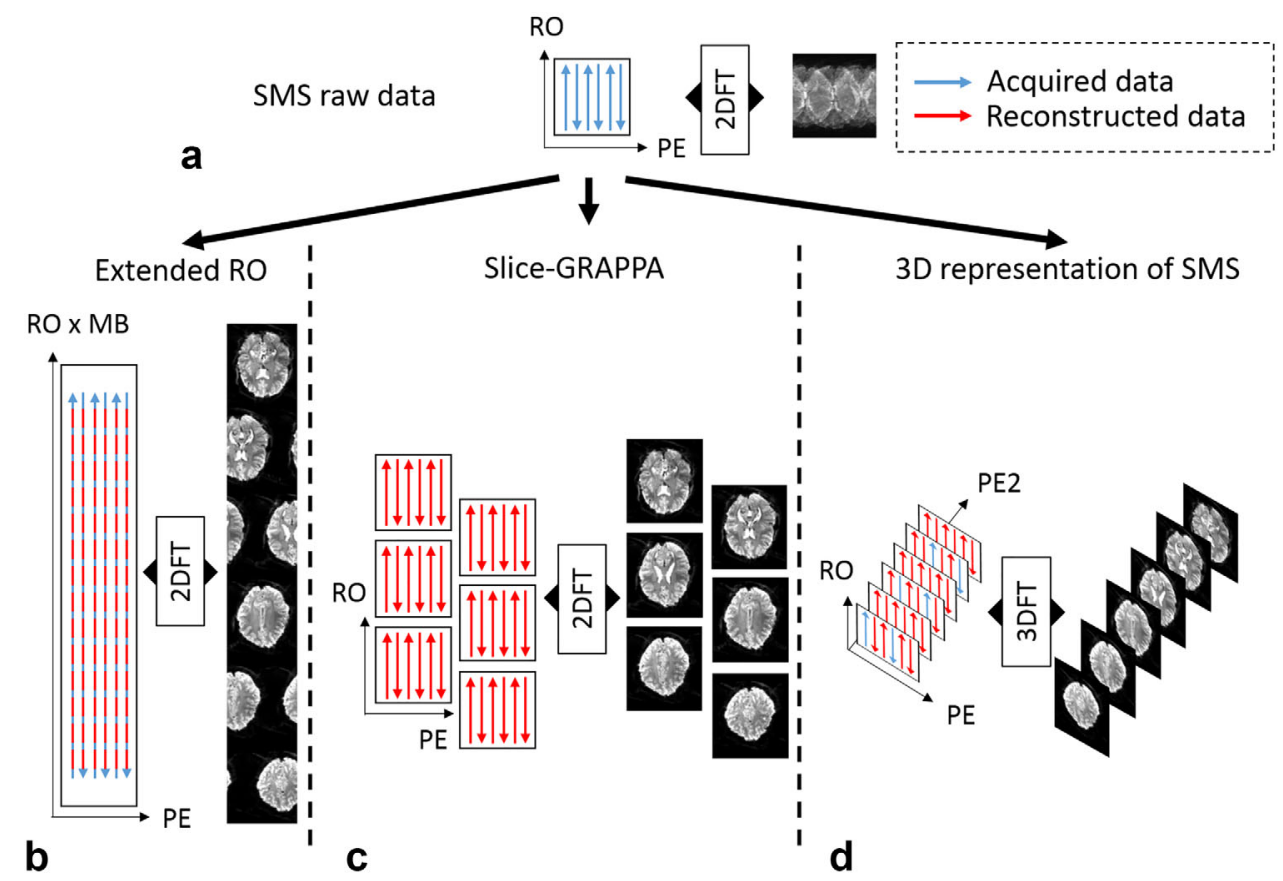

FIG. 2. (a) Undersampled MR data with SMS-EPI and controlled aliasing. (b) SMS slices (with shift $=F O V / 3$ ) are concatenated along the readout direction. GRAPPA is performed in this extended FOV where aliasing is equivalent to k-space undersampling. (c) Instead of filling out the missing part of k-space, the slice GRAPPA approach reconstructs a whole new k-space per slice. (d) SMS equivalent 3D representation, allowing SMS data to be reconstructed using 2D GRAPPA techniques as in Figure 1b. Blue and red lines indicate the acquired and reconstructed readout lines, respectively, for each method. 2DFT, 2D Fourier transform; 3DFT, 3D Fourier transform; GRAPPA, generalized autocalibrating partially parallel acquisition; $\mathrm{MB}$, multiband; $\mathrm{PE}$, phase-encoding; $\mathrm{RO}$, readout; $\mathrm{SMS}$, simultaneous multislice. 
and for 2D and 3D EPI sequences. The statistical significance of the results (between sequence/TR) was investigated using paired Student $t$ tests. A $P$ value of 0.05 was considered to indicate a significant difference.

To compare between reconstruction/sequences/TR, the fMRI data quality was estimated pre- and post-physiological noise correction using the temporal SNR normalized per unit time, defined for each voxel by $t S N R=\operatorname{mean}\left(S_{B O L D}(t)\right) /$ $\operatorname{std}\left(S_{B O L D}(t)\right) / \sqrt{T R}$ and averaged over all voxels within the gray matter (GM). Whole-brain normalized tSNR maps of 2D and 3D rsfMRI sequences were also compared at TR $=3300$ / 550/350 ms before and after physiological noise correction.

\section{Pre- and Postcorrection Resting State fMRI Performances}

Resting state networks (RSN) analysis was performed using two different approaches: independent component analysis (ICA) and seed-based correlation (SBC).

ICA was performed at subject level on uncorrected and corrected data using the melodic toolbox in FSL (38). After smoothing (6 mm kernel), voxel-wise mean removal, and variance normalization, the number of independent components was automatically estimated as described by Beckmann and Smith (39). For 2D and 3D EPI (TR=3300/550/ $350 \mathrm{~ms}$ ) this resulted in, on average, 35/180/400 and 30/ 150/350 components, respectively. After dimensionality reduction and ICA decomposition, the resulting networks were coregistered to the MNI space and compared with a 10-RSN atlas (40). Each atlas network was paired with the ICA component to which it was best spatially correlated. False-positive results (noisy/imperfect coregistration) were discarded manually. The results were compared using the following metrics: maximum Z-score for a specific RSN, and spatial correlation between the RSN and the atlas. Specifically, for each TR (350/550/3300 ms), the impact of sequence (2D/3D EPI), and physiological noise correction (uncorrected/corrected) was investigated using repeatedmeasures analysis of variance $(P<0.05)$.

SBC (41) was performed using the CONN toolbox (42) in MATLAB. After smoothing (6 mm kernel), coregistration (MNI template), and segmentation, rsfMRI data were cleaned from cerebrospinal fluid (CSF) and white matter (WM) contaminations. Three seeds (6 mm spheres) were used to investigate the following RSN: sensorimotor cortex, default mode network (DMN, seed in posterior cingulate cortex), and medial visual area. Correlation maps were derived at group level before and after physiological noise correction for the repetition times most affected by those nuisance signals ( $\mathrm{TR}=3300 / 550 \mathrm{~ms}$ ).

\section{RESULTS}

All three SMS reconstruction approaches were similarly affected by physiological noise (Supporting Fig. S1). tSNR improvements of $5 \%-6 \%$ were reached by removing RETc, RETr, CR, and RVT components from the 6fold accelerated data (Supporting Table S1). The 3D reconstruction resulted in lower tSNR in GM $(-10 \%$ / $-15 \%$ before/after correction compared with 2D EPI). Based on tSNR and reconstruction speed, accelerated 2D SMS data were reconstructed online using SG throughout the study.
Physiological regressors explained a small but significant part of the 2D EPI MR signal (Fig. 3a; $\mathrm{R}_{\text {adj }}^{2}=0.04-$ 0.07). $\mathrm{R}_{\mathrm{adj}}^{2}$ did not vary significantly with TR $(P>0.3)$. By comparison, physiological noise regressors explained a much larger signal fraction in unaccelerated segmented 3D EPI data (Fig. 3a; $R_{a d j}^{2}=0.35 \pm 0.11$ ). When using shorter TR and higher acceleration in 3D EPI, a substantial reduction in physiological noise was achieved compared with $\mathrm{TR}=3300 \mathrm{~ms} \quad\left(\mathrm{R}_{\text {adj }}^{2}=0.05-0.10\right.$ at $\mathrm{TR}=350 / 550 \mathrm{~ms}$; $P<0.05)$. Note that, at TR $=350 \mathrm{~ms}, 2 \mathrm{D}$ and 3D EPI sequences were similarly impacted by physiological signals $\left(R_{\text {adj }}^{2}=0.04-0.05\right)$, whose main contributors were cardiac RETROICOR (right panel of Fig. 3b, green, 70\%-78\% relative contribution), followed by respiratory RETROICOR (10\%-13\%). CR and RVT only resulted in minor signal contributions, especially for accelerated data $\left(R_{\text {adj }}^{2}<0.01\right)$.

Compared with 3D EPI (Fig. 3a, dashed bars), slow signal drifts explained a large portion of the 2D EPI data (plain bars in Fig. 3a; $\mathrm{R}_{\mathrm{adj}}^{2}=0.12 / 0.21 / 0.06$ for $\mathrm{TR}=3300$ / $550 / 350 \mathrm{~ms}$ ). Motion affected 2D and 3D EPI signal variance in a similar way $\left(\mathrm{R}_{\mathrm{adj}}^{2}=0.08-0.18\right)$.

The spatial contribution of the different nuisance signals is illustrated in Figure 4 on a representative subject (2D EPI, $\mathrm{TR}=550 \mathrm{~ms}$ ). Motion and slow signal drifts (Fig. 4a-d) mainly impacted posterior cortical areas. Respiration-based regressors (Fig. 4c-f) performed similarly on a much smaller scale and affected regions of high vessel density. On the other hand, cardiac RETROICOR and CR (Fig. 4b-e) mainly manifested themselves near large vessels.

This activity resulted in inhomogeneous tSNR increases within the brain when removing these signal fluctuations with hotspots near the ventricles and vasculature (Fig. 5). The overall impact of this correction remained modest, with the exception of 3D EPI at long sampling time (see black arrow in Fig. $5 \mathrm{a},+42 \%$ in GM, and up to $150 \%$ in lower brain regions most subject to physiological noise).

The only significant tSNR difference between 2D and 3D EPI data was found for TR $=3300 \mathrm{~ms}$ and without noise removal (Fig. 6a; $P=0.02$ ). Otherwise, the performances of $2 \mathrm{D}$ and $3 \mathrm{D}$ sequences were found remarkably consistent with each other for a given TR. Based on tSNR, there was a clear incentive to accelerate EPI data (TR $=550 \mathrm{~ms}$ versus $3300 \mathrm{~ms},+100 / 150 \%$ for 2D/3D EPI; $P=0.0002 / 0.0001)$. There was, however, no significant difference between 6and 8-fold accelerated data (TR 550 versus $350 \mathrm{~ms} ; P>0.8$ ), whether before or after physiological noise removal (Fig. $6 a, b)$.

Regarding ICA-derived RSNs, a substantial increase in Z-score was obtained by accelerating image acquisition for both 2D and 3D EPI $(+67 \% / 100 \%$ Z-score for SMS at 6/8-fold acceleration). For a given TR, analysis of variance yielded no significant effect of sequence type nor physiological noise removal on maximum Z-score and cross-correlation to the atlas $(P>0.05)$. Despite the significant tSNR increase with physiological noise removal, no changes were observed on the RSN extracted from ICA performed on 3D EPI data at long sampling time (Fig. 7a, TR $=3300 \mathrm{~ms}$ ). The complete correspondence between the ICA components best matching the atlas and the RSN atlas can be found in Supporting Figure S2.

SBC analysis (seeds location in Fig. 7b) revealed that physiological noise removal positively impacted 3D EPI 


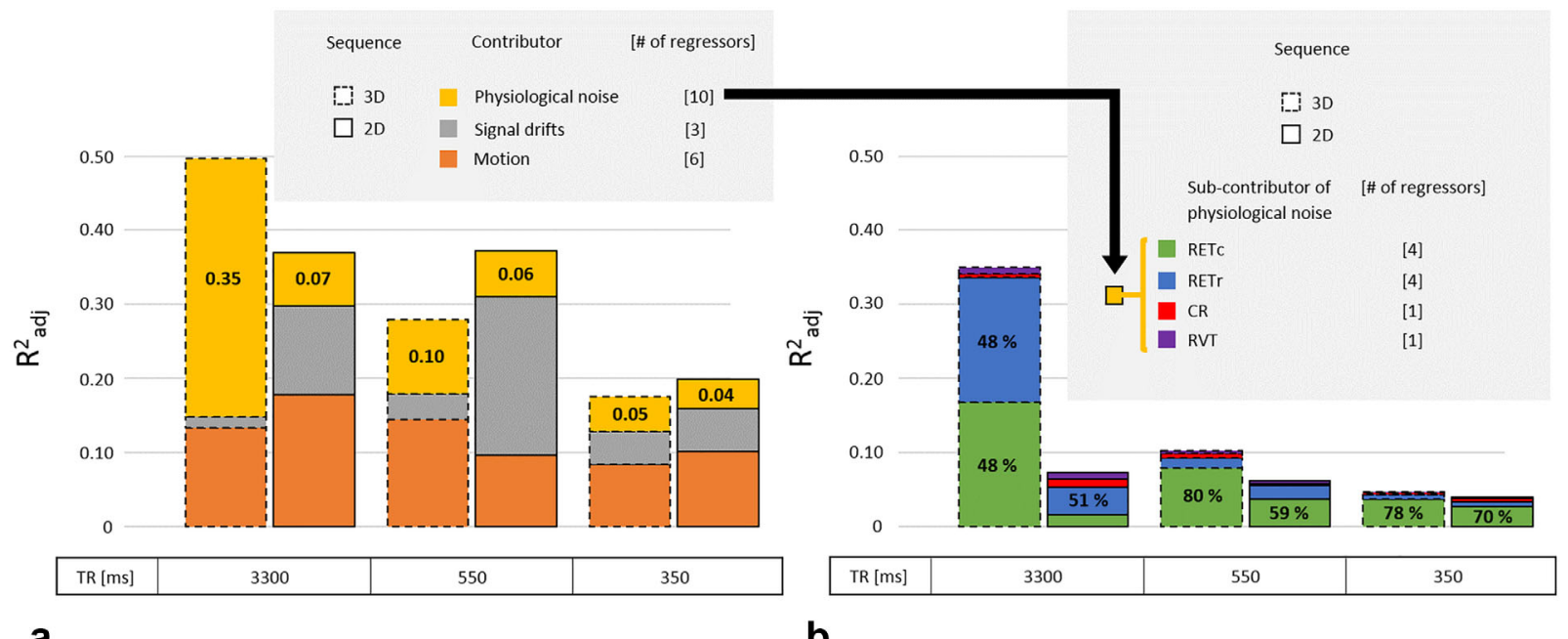

a

b

FIG. 3. (a) Normalized coefficient of determination $R_{\mathrm{adj}}^{2}$ illustrating the contribution of motion, signal drifts, and physiological signals to the fMRI signal acquired with various MR sequences (dashed/plain lines: 3D/2D-EPI) and repetition time (TR=3300/550/350 ms in left/ middle/right panel). For each component, the respective number of regressors is indicated within brackets. (b) Physiological noise (yellow in Fig. 3a) includes RETROICOR for cardiac (green) and respiration (blue), CR (red), and RVT (purple). For each sequence and TR, the relative contribution of the main physiological confounds is highlighted within the bar. CR, cardiac rate; RETc, cardiac RETROICOR; RETr, respiration RETROICOR; RVT, respiratory volume per unit time; TR, repetition time.

rsfMRI data by removing spurious correlation visible in $\mathrm{DMN} /$ medial visual area networks (see arrows in middle/bottom line). A few regions remained negatively correlated to the sensorimotor cortex seed after correction (top row). By contrast, differences due to noise removal were found negligible in 2D EPI for TR $=3300 \mathrm{~ms}$ (Fig. $7 \mathrm{~d}$ ) and for 2D and 3D rsfMRI data at shorter TR (data not shown). All other correlation maps, including 2D

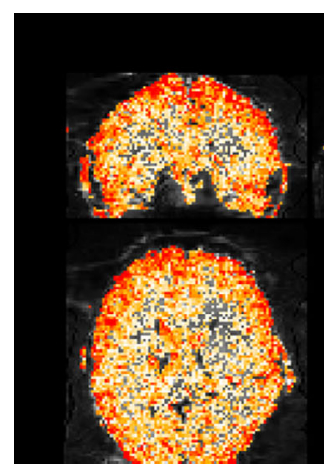

a
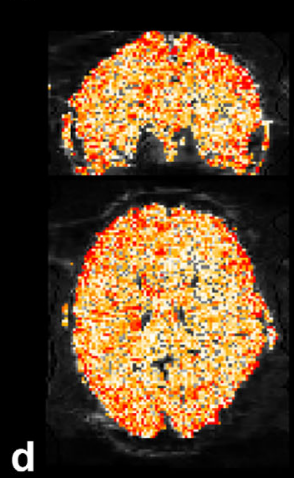

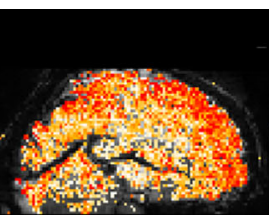

Motion [6]

\section{0}

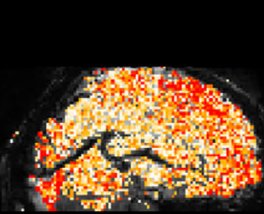

Polynoms [3]

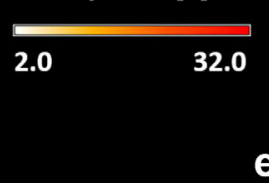

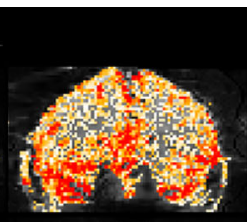
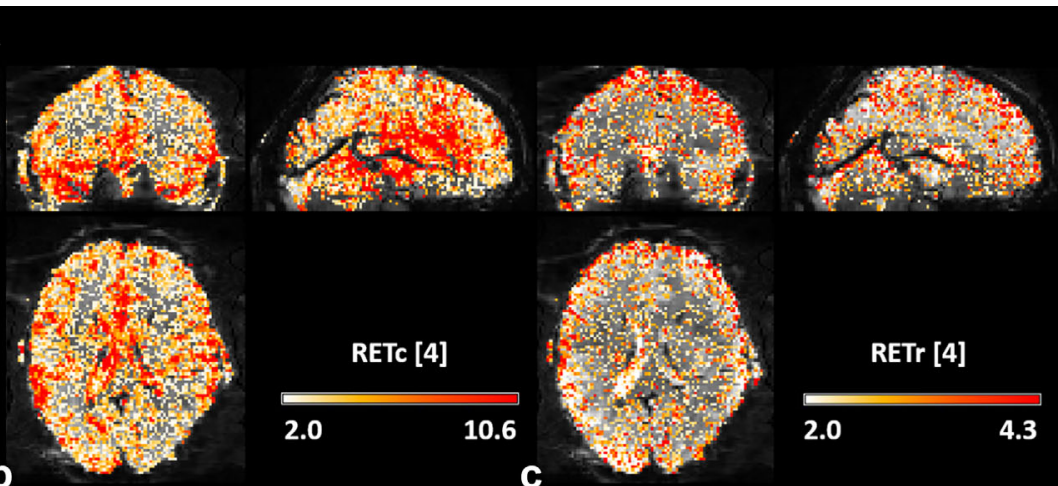

RETr [4]
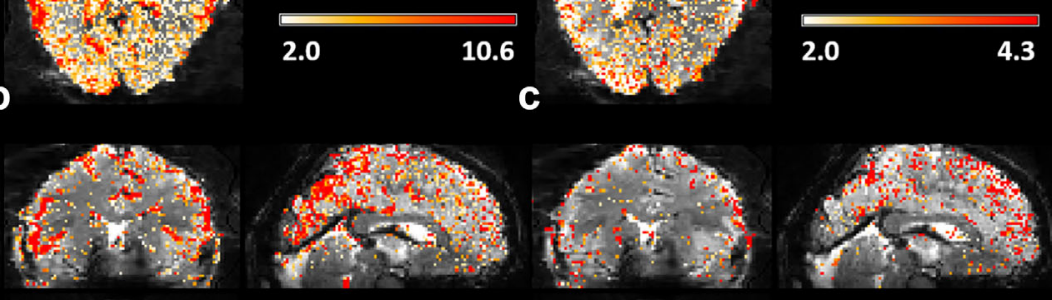

2.0

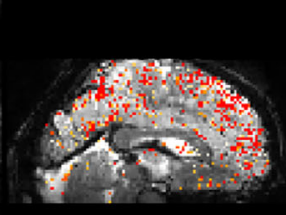

CR [1]
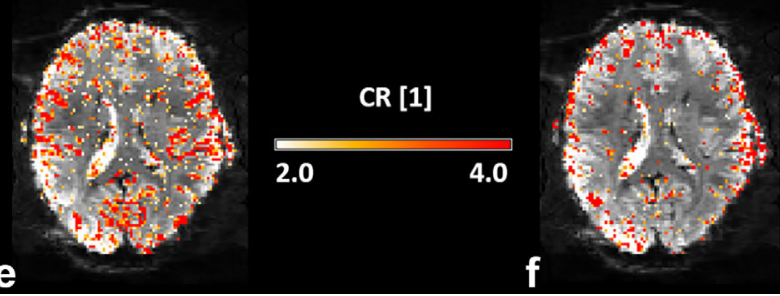

\section{RVT [1]}

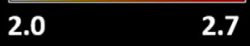

FIG. 4. Spatial contribution of the different nuisance signals on a representative subject (2D EPI, TR=550 ms). These maps represent, for each voxel, the maximum absolute value taken by the Z-score during the GLM analysis. For each panel, the number of corresponding time courses is indicated between brackets. Motion (a) and slow signal drifts (d, first, second, and third order polynomials) mainly impact posterior cortical areas, as do respiratory RETROICOR (c) and RVT (f) to a much lesser extent (note the scale difference between panels). Cardiac RETROICOR (b) and CR (e) manifest themselves near large vessels. CR, cardiac rate; RETc, cardiac RETROICOR; RETr, respiration RETROICOR; RVT, respiratory volume per unit time. 
Increase in temporal SNR due to physiological noise correction

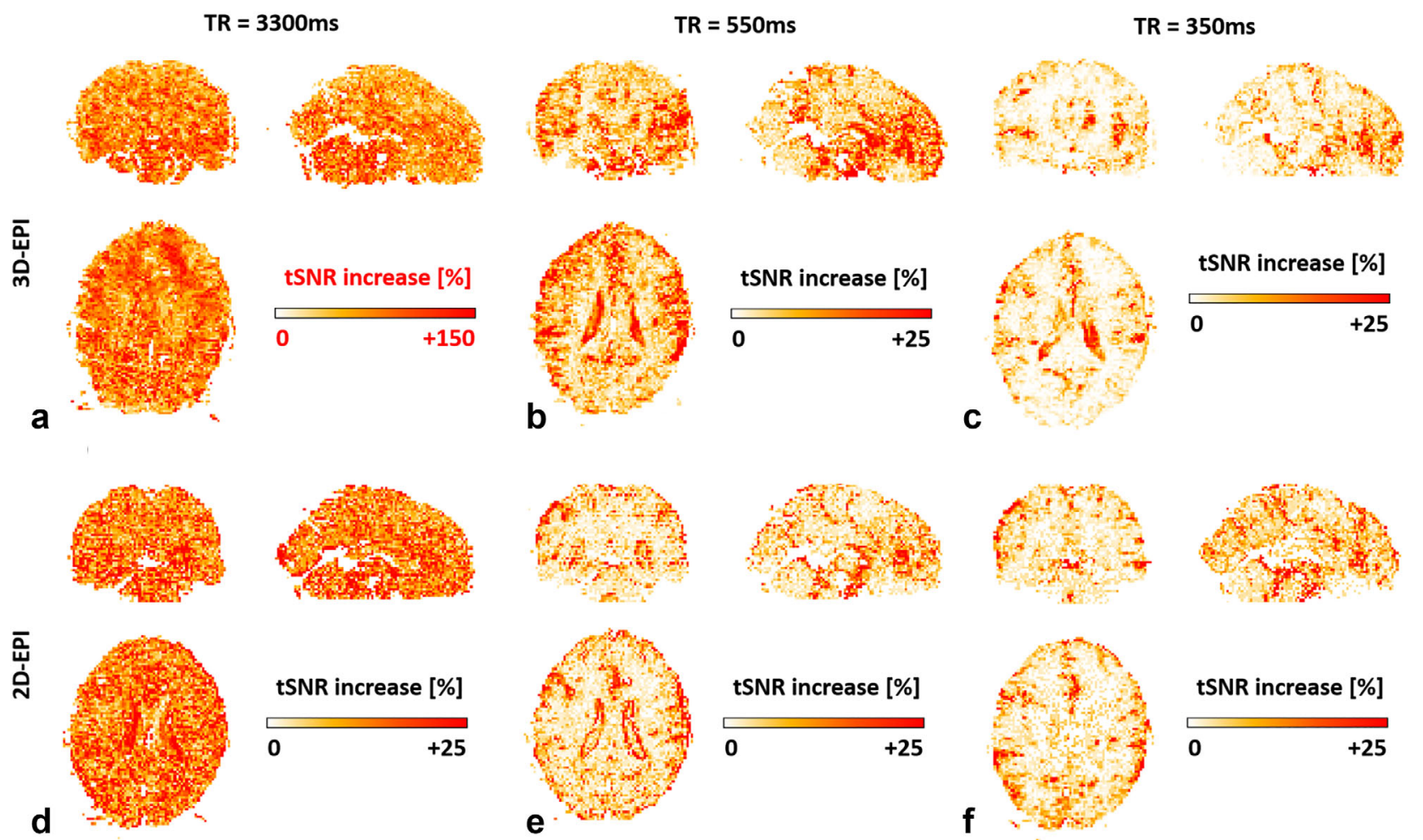

FIG. 5. tSNR increase due to physiological noise removal for 3D (a-c) and 2D (d-f) EPI and TR $=3300 / 550 / 350$ ms on a representative subject. Black arrows indicate the average tSNR increase in GM across subjects. Note the different scale in (a). EPI, echo planar imaging; TR, repetition time; tSNR, temporal signal-to-noise ratio.

\section{tSNR in GM}
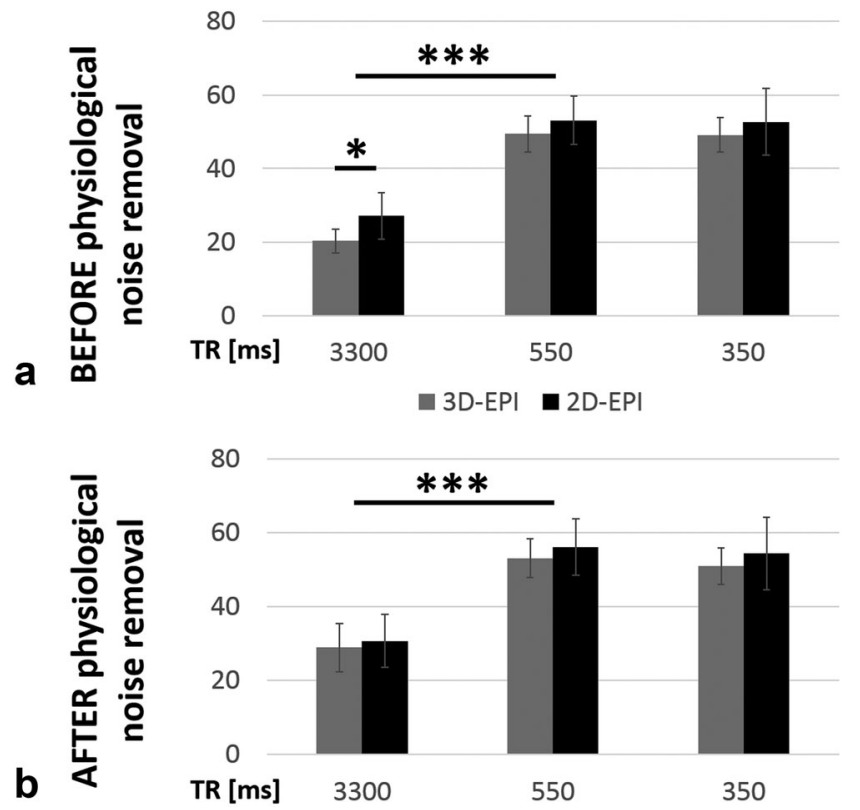

FIG. 6. Average tSNR in GM for 3D EPI (gray) and 2D EPI (black) before (a) and after (b) physiological noise correction $(n=8)$. Only six subjects were included for $\mathrm{TR}=3300 \mathrm{~ms}$. The only significant tSNR difference between 2D and 3D EPI was found for TR $=3300$ ms before noise removal $(P=0.02, \mathrm{n}=6)$. ${ }^{\star} P<0.05$. ${ }^{\star \star \star} P<0.001$. $\mathrm{EPI}$, echo planar imaging; GM, gray matter; TR, repetition time; tSNR, temporal signal-to-noise ratio. and 3D EPI at $\mathrm{TR}=550 \mathrm{~ms}$, were found overall to be cleaner than those derived for the long TR 3D EPI in Figure 7c, with increased positive correlation between posterior cingulate cortex, medial prefrontal cortex, and left/right angular gyri.

\section{DISCUSSION}

Physiological noise contributions to the total fMRI signal did not vary significantly with various SMS image reconstruction schemes, justifying the use of a single reconstruction approach for comparison with 3D techniques. Without acceleration, whole brain 3D techniques were found highly sensitive to physiological noise, as shown previously $(19,20)$. Compared with 2D EPI, they suffered from reduced tSNR and poor SBC-based rsfMRI performances when no correction was performed. Our results show that accelerated 3D EPI using controlled aliasing (18) with moderate acceleration factors $\left(R_{z}=6 / 8\right)$ were much less affected by physiological noise due to the shorter acquisition time and lower number of segments to be acquired (14). In this regimen, 2D and 3D techniques performed equally well, despite small tSNR differences that could be explained by the different image reconstruction approaches used in this study.

The age range of the subjects in this study is narrower than that used in most neuroscience fMRI studies. However, realignment estimates highlighted a very heterogeneous population from a motion perspective (root-mean-square of 


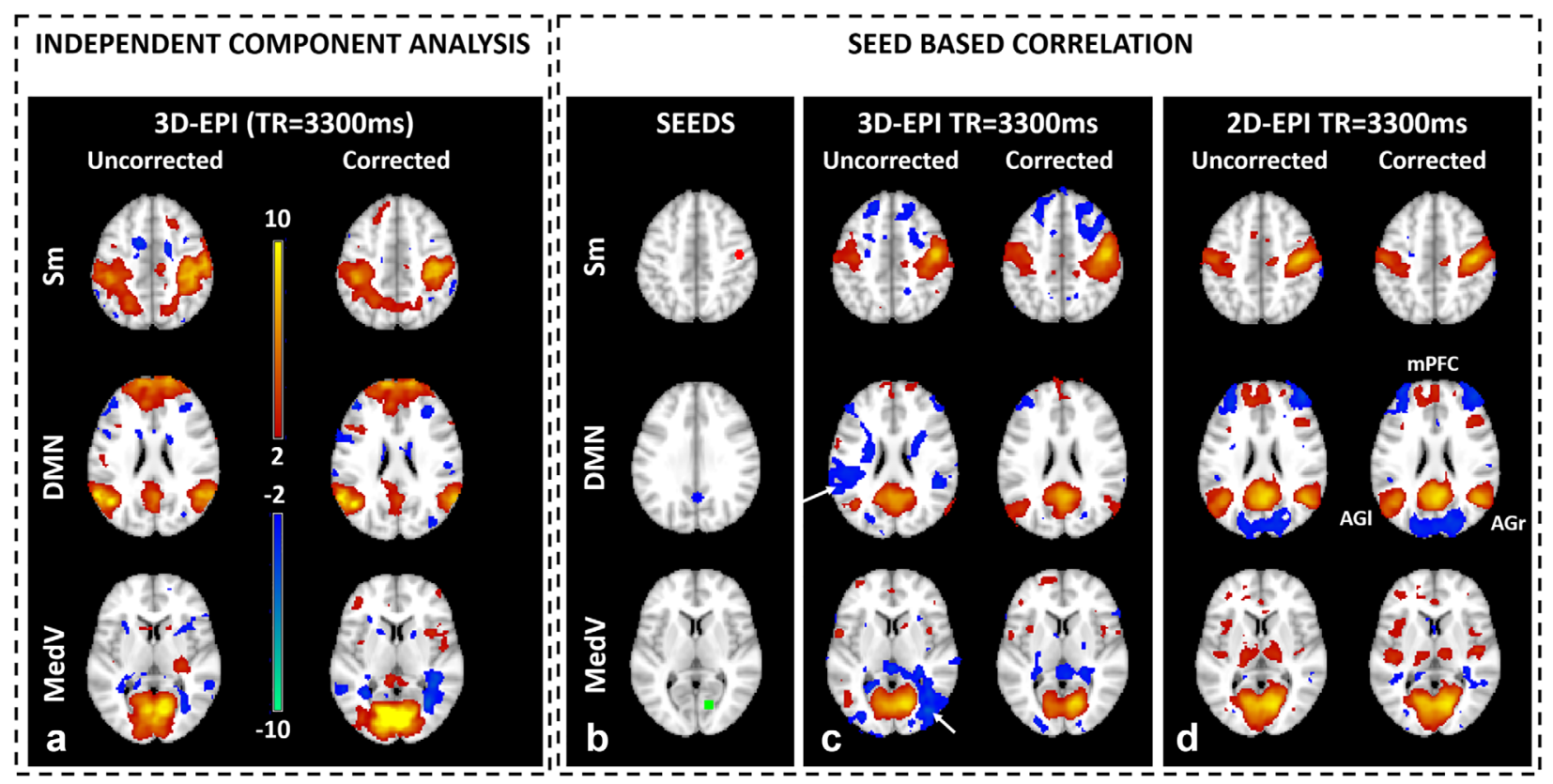

FIG. 7. (a) Group-level ICA analysis (temporal concatenation of 3D EPI data; $n=6$ ). Only three networks out of the 10-RSN atlas are illustrated here for clarity; the full correspondence with the atlas is provided in Supporting Figure S2. (b-d) Seed-based correlation results for $\mathrm{TR}=3300 \mathrm{~ms}$. (b) Location of seeds (6-mm spheres) in the right sensorimotor cortex (red), posterior cingulate cortex (blue), and right medial visual area (green). (c,d) Group level $(n=6)$ correlation maps (blue scale [-1.0:-0.2], red scale [0.2-1.0]) for 3D EPI (c) and 2D EPI (d) before and after physiological noise removal (left and right columns) in three representative slices. The white arrows indicate spurious correlation visible in DMN/medial visual area networks. AGL, left angular gyri; AGR, right angular gyri; DMN, default mode network; EPI, echo planar imaging; MedV, medial visual area; mPFC, medial prefrontal cortex; Sm, sensorimotor cortex; $T R$, repetition time.

translation in the range $0.10-1.33 \mathrm{~mm})$. Because other physiological nuisances such as respiration and cardiac rate only decrease mildly with age, we expect that these results can be extended to a wider population, more representative of a neuroscience study.

Amongst other limitations, this study focused on a single processing pipeline to model and estimate the physiological nuisance signals from the raw MR data and recorded respiration and cardiac cycle. The impact of the order of processing steps, between slice-timing correction and RETROICOR, was therefore not investigated. Although this might lead to suboptimal correction, studies have shown that the influence of this step only accounted for $0.3 \%$ of the signal variance (43). The cardiac and respiratory signals were convolved with the population-average response functions described by Chang et al. (44) and Brin et al. (27), respectively. Recent studies $(45,46)$ have suggested, however, that subject-specific transfer functions might remove additional signal noise. Future studies will investigate whether and how much this can benefit various sequences/ TR. Noise removal was performed in image space rather than k-space. For segmented 3D EPI, the validity of this approach was supported by previous work suggesting that k-space corrections do not outperform image-space corrections (21), allowing for a less computationally intensive approach and more direct comparison with 2D multislice sequences.

The choice of acceleration factors used in this study (1/6/8) covers a diverse range of relevant applications. Although nonaccelerated data are relevant for nonparallel imaging techniques, acceleration factors of 6 and 8 were deemed lossless and satisfactory, respectively, in terms of tSNR and image quality when using commercially available multiarray coils (32 channels in the present study).

The spatial contribution of the different nuisance signals was consistent in their physiological origins. In general, the effect of physiological noise was larger in GM than in WM (24). Respiration-based regressors impacted cortical areas and the vicinity of large/dense blood vessels, as reported by Brin et al. (26). Due to blood and CSF pulsatility, the effect of cardiac RETROICOR and CR was most apparent close to CSF and vessels such as the frontal and sagittal sinus, as reported by Glover et al. (22) and Dagli et al. (47).

The impact of physiological noise on 3D EPI data has been investigated previously (18-20). Our results differ from those of Jorge et al. (19) in that $3 \mathrm{D}$ sequences did not outperform 2D techniques after physiological noise removal. This difference can be explained by the fully optimized 2D EPI technique working with optimal flip angles. Lutti et al. (20) reported that the impact of physiological noise was estimated around $30 \%$, in agreement with our nonaccelerated 3D data. However, 3D techniques were found to be superior to $2 \mathrm{D}$ sequences in terms of tSNR $(+25 \%)$. Part of this discrepancy could be explained by the echo time difference reported by Lutti et al. (20), but the higher impact of physiological noise on 3D EPI at $7 \mathrm{~T}$ should not be disregarded. Although the ability to separate cardiac and respiration-induced artifacts has been evaluated by Narsude et al. (18), the added value of this work resides in the quantification and comparison of fully optimized 2D and 3D sequences, 
a necessary step to determine which sequence is best suited for fMRI at high fields.

$2 \mathrm{D}$ and $3 \mathrm{D}$ sequences were here compared at similar repetition times and acceleration factors, which resulted in fairly similar overall performances. Future studies could further investigate the flexibility offered by 3D accelerated sequences. Hybrid or more elaborate 3D encoding strategies, such as variable density k-space sampling (48), combined CAIPIRINHA and compressed sensing (49) or WAVE-CAIPIRINHA (50) may lead to increased tSNR for equal scan time, as shown at $3 \mathrm{~T}$ (51).

Despite numerous efforts in MRI acquisition, the direct comparison between 2D and 3D fMRI datasets is subject to two limitations: 1) within-session and subject variability and 2) different image reconstruction pipelines. Although counter-balancing runs helped mitigate the impact of the former, the latter was investigated using a single reconstruction pipeline for both techniques. In this study, the 2D SMS-EPI data acquired at TR $=550 \mathrm{~ms}$ were chosen to mimic the 3D k-space encoding used for 3D EPI. Similar acceleration factors were used $\left(\mathrm{MB}=\mathrm{R}_{\mathrm{z}}=6\right)$ and the SMS FOV shift and CAIPIRINHA shift adjusted using FO $V_{\text {shift }}=R_{z} / \Delta$ (33). Based on the similar k-space encoding pattern (illustrated in Figs. $1 \mathrm{~b}$ and $2 \mathrm{~d}$ ), both sequences could be reconstructed using 2D GRAPPA and similar kernels. However, k-space edges suffer with 2D GRAPPA from a reduced number of source points with conventional “optimized" GRAPPA kernels (Fig.1b). This has a large impact on SMS 3D-like reconstruction, because the extent of the SMS equivalent 3D k-space along PE2 equates the MB factor (Fig. 2d and Supporting Fig. S3). For the moderate acceleration factors used in practice $(\mathrm{MB}=3-8)$, edges can therefore account from one-quarter to two-thirds of k-space. More generally, for SMS-EPI the size of the GRAPPA kernel along PE2 is limited by the MB factor. Adapting GRAPPA kernels to cover k-space edges can help mitigate this effect (Supporting Fig. S3b), but results in suboptimal reconstructions for 3D-EPI data. For the purposes of this study, the reconstruction strategies chosen for SMS-EPI/3D-EPI were based on tSNR optimization rather than on a fully compatible processing pipeline.

Interestingly, tSNR-optimized $3 \mathrm{D}$ reconstructions for $2 \mathrm{D}$ and $3 \mathrm{D}$ data yielded very similar tSNR results. This might indicate that the reconstruction algorithms used in this study for 3D EPI could be improved, at least to the level of slice GRAPPA for SMS-EPI. Future work will investigate new methods of reconstructing accelerated 3D data. The acquisition of undersampled data might also benefit from recent advances in calibration line reordering, such as FLEET (52). Another issue lies in the potential trade-off between increased signal stability and image artifacts when using GRAPPA. Mean (i.e., time-averaged) volumes were visually inspected for image artifacts. Compared with fully sampled data, no apparent artifact resulted from the different reconstructions used in this manuscript.

In this study, small tSNR differences between 2D and 3D sequences did not result in different resting state fMRI sensitivity with ICA. Because of the ICA decomposition, spatially/temporally correlated noise that may affect tSNR is likely to be attributed to a specific component of no interest, whose contribution is effectively separated. As a result, fMRI performances or Z-scores in known networks can be less noise-dependent than Z-statistics in taskdriven fMRI, where the total signal variation is compared with a specific regressor and no ICA decomposition is performed. To verify this assumption, a similar analysis was performed here using seed-based correlation, as an intermediate step between task and rsfMRI. This resulted in improved correlation maps when the contribution of physiological noise was significant for nonaccelerated 3D EPI data. Although the extension of our results to task fMRI is immediate in terms of tSNR, the impact of physiological noise correction on fMRI sensitivity (activation maps, Zscores) is not straightforward. Unwanted correlation between the nuisance regressors and the stimulation paradigm will affect fMRI statistics, because noise removal is performed before statistical mapping. Any correlation between noise and task could effectively increase the rate of false-positives or false-negatives in a similar fashion. A full and comprehensive characterization of this effect would be stimulus-dependent (brain location, task type/ duration/frequency), thus falling outside the scope of this rsfMRI study.

In conclusion, physiological noise removal benefits rsfMRI performances at $7 \mathrm{~T}$. Nonaccelerated 3D EPI techniques with long sampling rates suffer the most from these nuisance signals. For moderately high acceleration factors, 3D EPI sequences provide equal tSNR compared with SMS-EPI when using optimized image reconstruction and physiological noise removal. Both techniques can be used at $7 \mathrm{~T}$ with equal rsfMRI performances.

\section{ACKNOWLEDGMENTS}

We thank Dr. Mayur Narsude for preliminary studies and sharing code for data processing.

\section{REFERENCES}

1. Mansfield P. Multi-planar image formation using NMR spin echoes. J Phys C Solid State Phys 1977;10:L55-L58.

2. Setsompop K, Feinberg DA, Polimeni JR. Rapid brain MRI acquisition techniques at ultra-high fields. NMR Biomed 2016;29:1198-1221.

3. Sodickson DK, Manning WJ. Simultaneous acquisition of spatial harmonics (SMASH): fast imaging with radiofrequency coil arrays. Magn Reson Med 1997;38:591-603.

4. Griswold MA, Jakob PM, Heidemann RM, Nittka M, Jellus V, Wang J, Kiefer B, Haase A. Generalized autocalibrating partially parallel acquisitions (GRAPPA). Magn Reson Med 2002;47:1202-1210.

5. Pruessmann KP, Weiger M, Scheidegger MB, Boesiger P. SENSE: sensitivity encoding for fast MRI. Magn Reson Med 1999;42:952-962.

6. Larkman DJ, Hajnal JV, Herlihy AH, Coutts GA, Young IR, Ehnholm G. Use of multicoil arrays for separation of signal from multiple slices simultaneously excited. J Magn Reson Imaging 2001;13:313-317.

7. Nunes RG, Hajnal JV, Golay X, Larkman DJ. Simultaneous Slice Excitation and Reconstruction for Single Shot EPI. In: Proceedings of the 14th Annual Meeting of ISMRM, Seattle, Washington, USA, 2006. p. 293.

8. Setsompop K, Gagoski BA, Polimeni JR, Witzel T, Wedeen VJ, Wald LL. Blipped-controlled aliasing in parallel imaging for simultaneous multislice echo planar imaging with reduced g-factor penalty. Magn Reson Med 2012;67:1210-1224.

9. Moeller S, Yacoub E, Olman CA, Auerbach E, Strupp J, Harel N, Ugurbil K. Multiband multislice GE-EPI at 7 Tesla, with 16 -fold acceleration using partial parallel imaging with application to high spatial and temporal whole-brain FMRI. Magn Reson Med 2010;63: 1144-1153.

10. Feinberg DA, Moeller S, Smith SM, Auerbach E, Ramanna S, Glasser MF, Miller KL, Ugurbil K, Yacoub E. Multiplexed echo planar imaging for sub-second whole brain fMRI and fast diffusion imaging. PLoS One 2010;5:e15710. 
11. Mansfield P, Coxon R, Hykin J. Echo-volumar imaging (EVI) of the brain at 3.0 T: first normal volunteer and functional imaging results. J Comput Assist Tomogr 1995;19:847-852.

12. Van Der Zwaag W, Francis S, Bowtell R. Improved echo volumar imaging (EVI) for functional MRI. Magn Reson Med 2006;56:1320-1327.

13. Poser BA, Koopmans PJ, Witzel T, Wald LL, Barth M. Three dimensional echo-planar imaging at 7 Tesla. Neuroimage 2010;51:261-266.

14. Van Der Zwaag W, Marques JP, Kober T, Glover G, Gruetter R, Krueger G. Temporal SNR characteristics in segmented 3D-EPI at 7T. Magn Reson Med 2012;67:344-352.

15. Lai S, Glover GH. Three-dimensional spiral fMRI technique: a comparison with 2D spiral acquisition. Magn Reson Med 1998;39:68-78.

16. Hu Y, Glover GH. Three-dimensional spiral technique for highresolution functional MRI. Magn Reson Med 2007;58:947-951.

17. Friston KJ, Williams S, Howard R, Frackowiak RS, Turner R. Movement-related effects in fMRI time-series. Magn Reson Med 1996;35: 346-355.

18. Narsude M, Gallichan D, van der Zwaag W, Gruetter R, Marques JP. Three-dimensional echo planar imaging with controlled aliasing: a sequence for high temporal resolution functional MRI. Magn Reson Med 2016;75:2350-2361.

19. Jorge J, Figueiredo P, van der Zwaag W, Marques JP. Signal fluctuations in fMRI data acquired with 2D-EPI and 3D-EPI at 7 Tesla. Magn Reson Imaging 2013;31:212-220.

20. Lutti A, Thomas DL, Hutton C, Weiskopf N. High-resolution functional MRI at $3 \mathrm{~T}$ : 3D/2D echo-planar imaging with optimized physiological noise correction. Magn Reson Med 2013;69:1657-1664.

21. Tijssen RHN, Jenkinson M, Brooks JCW, Jezzard P, Miller KL. Optimizing RetroICor and RetroKCor corrections for multi-shot 3D FMRI acquisitions. Neuroimage 2014;84:394-405.

22. Glover GH, Li TQ, Ress D. Image-based method for retrospective correction of physiological motion effects in fMRI: RETROICOR. Magn Reson Med 2000;44:162-167.

23. Bianciardi M, Fukunaga M, van Gelderen P, Horovitz SG, de Zwart JA, Shmueli K, Duyn JH. Sources of functional magnetic resonance imaging signal fluctuations in the human brain at rest: a $7 \mathrm{~T}$ study. Magn Reson Imaging 2009;27:1019-1029.

24. Chang C, Glover GH. Relationship between respiration, end-tidal CO2, and BOLD signals in resting-state fMRI. Neuroimage 2009;47:1381-1393.

25. Shmueli K, van Gelderen P, de Zwart JA, Horovitz SG, Fukunaga M, Jansma JM, Duyn JH. Low-frequency fluctuations in the cardiac rate as a source of variance in the resting-state fMRI BOLD signal. Neuroimage 2007;38:306-320.

26. Birn RM, Diamond JB, Smith MA, Bandettini PA. Separating respiratory-variation-related fluctuations from neuronal-activityrelated fluctuations in fMRI. Neuroimage 2006;31:1536-1548.

27. Birn RM, Smith MA, Jones TB, Bandettini PA. The respiration response function: the temporal dynamics of fMRI signal fluctuations related to changes in respiration. Neuroimage 2008;40:644-654.

28. Hutton C, Josephs O, Stadler J, Featherstone E, Reid A, Speck O, Bernarding J, Weiskopf N. The impact of physiological noise correction on fMRI at 7T. Neuroimage 2011;57:101-112.

29. van der Zwaag W, Jorge J, Butticaz D, Gruetter R. Physiological noise in human cerebellar fMRI. MAGMA 2015;28:485-492.

30. Krüger G, Glover GH. Physiological noise in oxygenation-sensitive magnetic resonance imaging. Magn Reson Med 2001;46:631-637.

31. Blaimer M, Breuer FA, Seiberlich N, Mueller MF, Heidemann RM, Jellus V, Wiggins G, Wald LL, Griswold MA, Jakob PM. Accelerated volumetric MRI with a SENSE/GRAPPA combination. J Magn Reson Imaging 2006;24:444-450.

32. Cauley SF, Polimeni JR, Bhat H, Wald LL, Setsompop K. Interslice leakage artifact reduction technique for simultaneous multislice acquisitions. Magn Reson Med 2014;72:93-102.

33. Zahneisen B, Ernst T, Poser BA. SENSE and simultaneous multislice imaging. Magn Reson Med 2015;74:1356-1362.

34. Zahneisen B, Poser BA, Ernst T, Stenger VA. Three-dimensional Fourier encoding of simultaneously excited slices: generalized acquisition and reconstruction framework. Magn Reson Med 2014;71:2071-2081.

35. Moeller S, Vu AT, Auerbach E, Ugurbil K, Yacoub E. RO extended FOV SENSE/GRAPPA for multiband imaging with FOV shift. In Proceedings of the 22nd Annual Meeting of ISMRM, Milan, Italy, 2014. p. 4396.

36. Salomon R, Darulova J, Narsude M, Van Der Zwaag W. Comparison of an 8-channel and a 32-channel coil for high-resolution fMRI at $7 \mathrm{~T}$. Brain Topogr 2014;27:209-212.
37. Breuer FA, Blaimer M, Mueller MF, Seiberlich N, Heidemann RM, Griswold MA, Jakob PM. Controlled aliasing in volumetric parallel imaging (2D CAIPIRINHA). Magn Reson Med 2006;55:549-556.

38. Smith SM, Jenkinson M, Woolrich MW, et al. Advances in functional and structural MR image analysis and implementation as FSL. NeuroImage 2004;23(Suppl 1):S208-S219.

39. Beckmann CF, Smith SM. Probabilistic independent component analysis for functional magnetic resonance imaging. IEEE Trans Med Imaging 2004;23:137-152.

40. Smith SM, Fox PT, Miller KL, et al. Correspondence of the brain's functional architecture during activation and rest. Proc Natl Acad Sci U S A 2009;106:13040-13045.

41. Biswal B, Yetkin FZ, Haughton VM, Hyde JS. Functional connectivity in the motor cortex of resting human brain using echo-planar MRI. Magn Reson Med 1995;34:537-541.

42. Whitfield-Gabrieli S, Nieto-Castanon A. Conn: a functional connectivity toolbox for correlated and anticorrelated brain networks. Brain Connect 2012;2:125-141.

43. Jones TB, Bandettini PA, Birn RM. Integration of motion correction and physiological noise regression in fMRI. Neuroimage 2008;42:582-590.

44. Chang C, Cunningham JP, Glover GH. Influence of heart rate on the BOLD signal: the cardiac response function. Neuroimage 2009;44:857-869.

45. Falahpour M, Refai H, Bodurka J. Subject specific BOLD fMRI respiratory and cardiac response functions obtained from global signal. Neuroimage 2013;72:252-264.

46. Golestani AM, Chang C, Kwinta JB, Khatamian YB, Jean Chen J. Mapping the end-tidal CO2 response function in the resting-state BOLD fMRI signal: spatial specificity, test-retest reliability and effect of fMRI sampling rate. Neuroimage 2015;104:266-277.

47. Dagli MS, Ingeholm JE, Haxby J V. Localization of cardiac-induced signal change in fMRI. Neuroimage 1999;9:407-415.

48. Tsai CM, Nishimura DG. Reduced aliasing artifacts using variable-density k-space sampling trajectories. Magn Reson Med 2000;43:452-458.

49. Stäb D, Wech T, Breuer FA, Weng AM, Ritter CO, Hahn D, Köstler H. High resolution myocardial first-pass perfusion imaging with extended anatomic coverage. J Magn Reson Imaging 2014;39:1575-1587.

50. Bilgic B, Gagoski BA, Cauley SF, Fan AP, Polimeni JR, Grant PE, Wald LL, Setsompop K. Wave-CAIPI for highly accelerated 3D imaging. Magn Reson Med 2015;73:2152-2162.

51. Stirnberg R, Huijbers W, Poser BA, Stocker T. Ultra-fast gradient echo EPI with controlled aliasing at 3T: simultaneous multi-slice vs. 3D-EPI. In Proceedings of the 24th Annual Meeting of ISMRM, Singapore, 2016. p. 941.

52. Polimeni JR, Bhat H, Witzel T, Benner T, Feiweier T, Inati SJ, Renvall V, Heberlein K, Wald LL. Reducing sensitivity losses due to respiration and motion in accelerated echo planar imaging by reordering the autocalibration data acquisition. Magn Reson Med 2016;75:665-679.

\section{SUPPORTING INFORMATION}

Additional Supporting Information may be found in the online version of this article.

Fig. S1. Improvement in adjusted coefficient of determination $R_{a d j}^{2}$ due to partial physiological noise removal after different SMS reconstructions $(\mathrm{MB}=6, \mathrm{TR}=550 \mathrm{~ms}$ ). The extended readout (orange), slice GRAPPA (blue), and 3D reconstruction approaches (gray) are illustrated in Figure 1C, $1 \mathrm{D}$, and $1 \mathrm{E}$, respectively. No significant difference was found between the three reconstructions $(P>0.05)$.

Fig. S2. Full correspondence between the $10-\mathrm{RSN}$ atlas (40) and the 10 best matching components from the group-level ICA for 3D EPI rsfMRI data $(T R=3300 \mathrm{~ms}$, top row). The Z-score scales in [2-10] (red/blue, positive/negative). From left to right, the networks of reference are: medial visual (MedV); occipital visual (OccV); lateral visual (LatV); default mode network (DMN); cerebellum (Cb); sensorimotor (Sm); auditory (aud); executive control (ExC); and left and right fronto-parietal lobes (FP1/FP2).

Fig. S3. tSNR optimized GRAPPA kernels used for reconstruction of (A) 3D EPI data and (B) SMS-EPI data with 6-fold acceleration. Target points (gray) are reconstructed based on neighboring acquired MR data (source points, black circles). The kernel is shifted along $k_{\mathrm{PE}}$ and $\mathrm{k}_{\mathrm{PE} 2}$ to reconstruct the whole k-space. For SMS-EPI, the full extent of the 3D equivalent k-space is limited by the MB factor. Optimized image reconstruction requires modification of the GRAPPA kernel to incorporate k-space edges.

Table S1. Impact of motion correction, slow signal drifts, and physiological noise removal on tSNR per unit time in gray matter for the various SMS reconstructions illustrated in Figure 2 , and $\mathrm{TR}=550 \mathrm{~ms}(\mathrm{MB}=6)$. 